\title{
REVIEW
}

\section{Alogliptin-Pioglitazone Combination Therapy: A Rational Approach to Treating Type 2 Diabetes Mellitus}

Kohei Kaku · Masashi Hirayama · Emiko Komura

To view enhanced content go to www.combitherapy-open.com Received: July 31, 2012 / Published online: October 3, 2012

(c) The Author(s) 2012. This article is published with open access at Springerlink.com

\section{ABSTRACT}

Introduction: Type 2 diabetes mellitus (T2DM) is a complex disease with a number of metabolic abnormalities. At present, treatment typically proceeds in a stepwise fashion, beginning with diet and exercise followed by incremental additions of oral antidiabetic agents as required to achieve and maintain glycemic control (glycosylated hemoglobin $\left[\mathrm{HbA}_{1 \mathrm{c}}\right] \leq 6.9 \%$ and $6.5 \%$, respectively). This approach is reactive rather than proactive as progression to the next level is based on treatment failure (i.e., not achieving target $\mathrm{HbA}_{1 \mathrm{c}}$ levels). Newer approaches

\section{K. Kaku ( $\square)$}

Division of Diabetes, Endocrinology and Metabolism,

Department of Medicine, Kawasaki Medical School,

Okayama, Japan

e-mail: kka@med.kawasaki-m.ac.jp

M. Hirayama · E. Komura

Pharmaceutical Development Division, Takeda

Pharmaceutical Company Ltd, Osaka, Japan

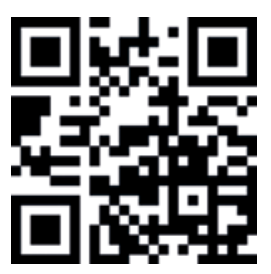

Enhanced content for this article is available on the journal web site: www.combitherapy-open.com to treatment of T2DM advocate early use of combination antihyperglycemic regimens with complementary mechanisms of action to correct multiple pathophysiologic defects, but this can impact negatively on treatment adherence. Fixed-dose combinations are associated with higher compliance rates than therapy with individual components administered concomitantly. This review examines evidence for a fixed-dose combination of alogliptin and pioglitazone recently approved for use in Japanese patients with T2DM.

Methods: A MEDLINE search identified five randomized, controlled trials in which alogliptin and pioglitazone were used in combination to treat T2DM, and these form the basis of the review. Results: One study evaluated alogliptinpioglitazone combination therapy in drug-naïve patients. In the remaining studies, alogliptin was evaluated as add-on therapy in patients with inadequate glycemic control despite treatment with pioglitazone $( \pm$ metformin or sulfonylurea). In all studies, alogliptinpioglitazone combination therapy consistently produced statistically significant reductions in $\mathrm{HbA}_{1 \mathrm{c}}$ of approximately $0.6-1.0 \%$ over and above those produced by either agent alone. This improvement was paralleled by improvements in 
fasting plasma glucose, proportions of patients achieving target $\mathrm{HbA}_{1 \mathrm{c}}$ levels and several other measures of glycemic control, including markers of beta-cell function. The alogliptin-pioglitazone combination was well tolerated across all studies. Conclusion: Alogliptin-pioglitazone combination therapy represents a rational approach to treatment of $\mathrm{T} 2 \mathrm{DM}$ as the complementary mechanisms target several aspects of impaired glucose control. The fixed-dose combination offers scope for enhanced patient compliance and improved treatment outcomes.

Keywords: Alogliptin; Beta-cell function; Combination therapy; Fixed-dose combinations; Glycosylated hemoglobin; Pioglitazone; Type 2 diabetes mellitus

\section{INTRODUCTION}

Type 2 diabetes mellitus (T2DM) is a disorder of blood glucose control that arises from multiple metabolic abnormalities: impaired response of liver and peripheral (muscle, adipose) tissues to the effects of insulin (i.e., insulin resistance); progressive loss of pancreatic beta-cell function (insufficient insulin secretion, reduced beta-cell sensitivity to glucose, decreased beta-cell mass); dysregulation of glucagon secretion; and altered incretin hormone physiology (Fig. 1) [1]. These defects all lead to high levels of postprandial and fasting blood glucose.

Early and effective treatment of T2DM is essential to minimize the microvascular and macrovascular complications associated with chronic hyperglycemia, but success remains limited. Only about half of patients with T2DM in the United States (US) achieve the American Diabetes Association glycosylated hemoglobin $\left(\mathrm{HbA}_{1 \mathrm{c}}\right)$ goal of $<7.0 \%$ [2] and the proportion decreases as the $\mathrm{HbA}_{1 \mathrm{c}}$ target becomes more aggressive. A cross-sectional survey involving $>17,000$ patients in Japan found that just over one-third (36.8\%) achieved the $\mathrm{HbA}_{1 \mathrm{c}}$ target goal of $<6.9 \%$ as recommended by the Japan Diabetes Society [3].

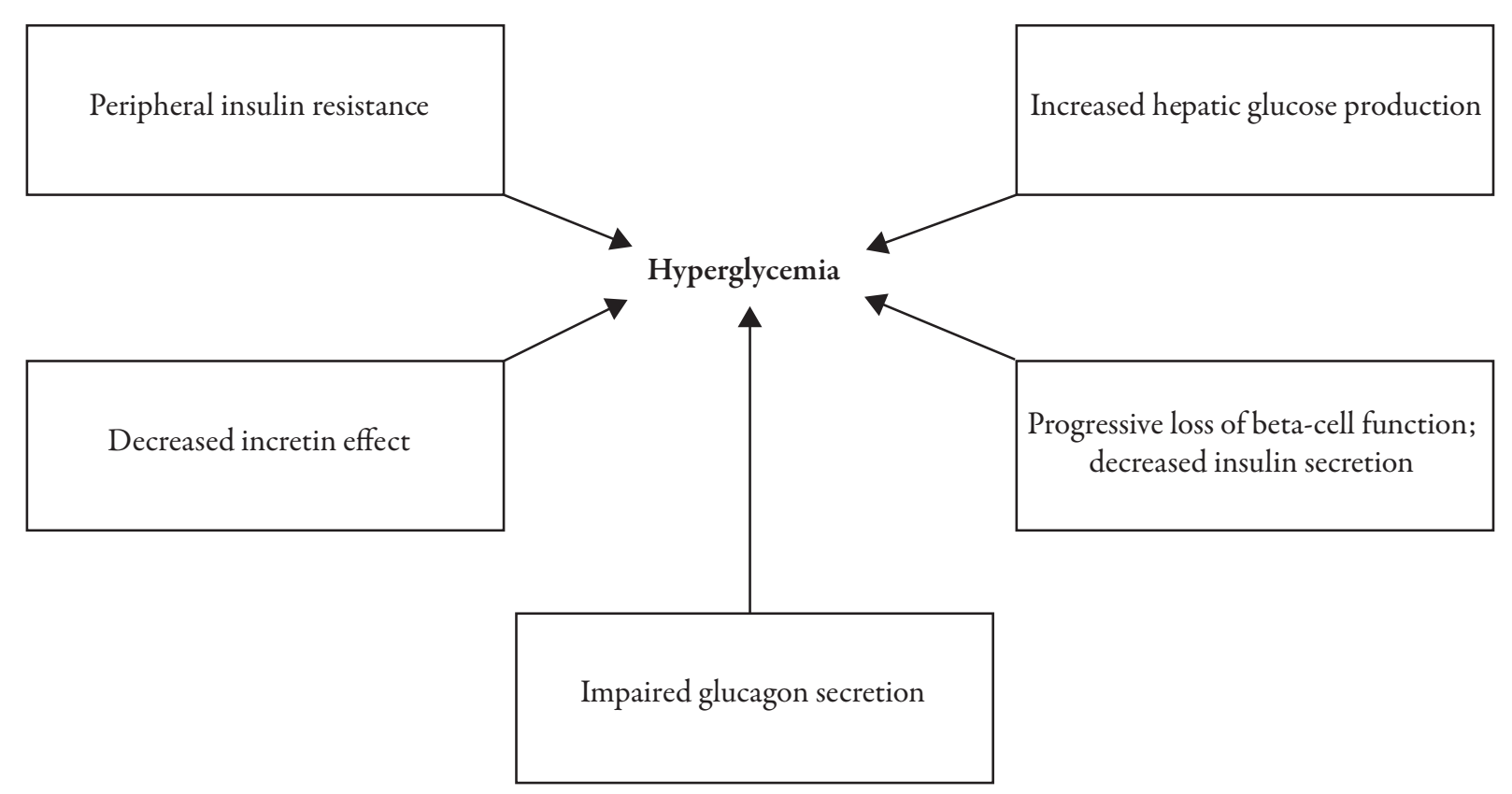

Fig. 1 Major pathophysiological features of type 2 diabetes mellitus 
Treatment of T2DM typically begins with diet/exercise followed by incremental additions of single antidiabetic agents as required, most commonly metformin or a sulfonylurea to achieve and maintain glycemic control. This approach is reactive rather than proactive as progression to the next level is based on treatment failure. Treatment by single oral agents are unable to address all the metabolic abnormalities that underlie T2DM and the ability to treat aggressively is often limited by dosedependent side effects [4]. As T2DM progresses, most patients require the addition of one or more antidiabetic agents to regain glycemic control. Combining agents with complementary mechanisms of action is a rational approach to addressing multiple defects in glycemic control with the potential of achieving additive clinical benefits [5]. However, multi-drug therapy can have a negative impact on patient adherence to treatment, particularly in the case of chronic diseases, such as T2DM [6-9]. Accordingly, interest is growing in the use of fixed-dose combinations of antidiabetic agents which are not only more convenient for patients, but also have the potential to improve adherence [10]. Many such combinations are possible, but one of considerable interest involves the dipeptidyl peptidase-4 (DPP-4) inhibitor and thiazolidinedione (TZD) classes of oral antidiabetic agents. DPP-4 inhibitors (or 'gliptins') act primarily by increasing pancreatic insulin secretion in response to nutrient intake [11, 12], whereas TZDs act predominantly by improving the sensitivity of muscle and adipose tissue to insulin [13]. By addressing two of the fundamental defects in T2DM - namely, beta-cell dysfunction and insulin resistance - this combination offers scope for additive efficacy.

An alogliptin-pioglitazone fixed-dose combination tablet was recently approved in
Japan for use in patients with T2DM. This review examines in more detail the pharmacologic rationale for combining DPP-4 inhibitors and TZDs. In particular, this review focuses on clinical trials in which alogliptin and pioglitazone have been used in combination for the treatment of Japanese patients with T2DM.

\section{METHODS}

A MEDLINE search was performed using the key words "alogliptin" and "pioglitazone" to identify relevant articles for inclusion. A total of five randomized, controlled clinical studies of alogliptin-pioglitazone combination therapy up to and including May 2012 were identified and form the basis of this review. These studies were supplemented by major reviews and other important articles known to the authors.

\section{PHARMACOLOGICAL RATIONALE FOR ALOGLIPTIN-PIOGLITAZONE COMBINATION}

\section{Pioglitazone}

Pioglitazone is a potent insulin sensitizer. It binds specifically to the peroxisome proliferatoractivated receptor gamma (PPARG) and enhances the sensitivity of liver, muscle, and adipose tissue to insulin (promoting the uptake of glucose into tissues), with a resulting decline in fasting and postprandial plasma glucose levels [14, 15]. Pioglitazone also increases serum high-density lipoprotein (HDL)-cholesterol and decreases neutral fat [13], whilst increasing the expression and differentiation of adiponectin, a hormone that enhances insulin sensitivity $[16,17]$. Several long-term (>1.5 years), double-blind, active comparator studies have consistently demonstrated that pioglitazone produces a durable decrease in $\mathrm{HbA}_{1 \mathrm{c}}$ levels [18-22], 
a result which can be explained only by its ability to improve and preserve beta-cell function in patients with T2DM [23]. In other studies involving patients with T2DM, pioglitazone was shown to reduce the inflammatory response to a glucose challenge more than acarbose [24] and it was shown to be better than glibenclamide in mitigating variations in lipid parameters and inflammatory markers [25].

Pioglitazone is well absorbed after oral administration and reaches maximum concentrations in approximately $1.5 \mathrm{~h}$ [26]. Although metabolized by the hepatic cytochrome P450 enzyme system, there is no evidence to suggest that pioglitazone inhibits or induces any of the $\mathrm{P} 450$ isoenzymes involved in drug metabolism [27, 28]. The half-life of pioglitazone is approximately $9 \mathrm{~h}$, but its glucose-lowering activity is extended by two active metabolites. The pharmacokinetic characteristics of pioglitazone are not significantly altered in patients with T2DM, renal or hepatic insufficiency, or in the elderly. Pioglitazone can be administered once daily without regard to meals [26].

\section{Alogliptin}

Alogliptin is a potent and highly selective inhibitor of DPP-4, the enzyme responsible for rapid inactivation of the incretin hormones, glucagon-like peptide-1 (GLP-1) and glucose dependent insulinotropic polypeptide (GIP) [12]. GLP-1 and GIP are secreted in the intestine in response to food intake and stimulate insulin secretion from pancreatic beta cells in a glucosedependent manner. GLP-1 also suppresses glucagon secretion and enhances pancreatic beta-cell mass through increases in proliferation and decreases in apoptosis [1]. GLP-1 has been shown to lower glucose levels even in patients with severe beta-cell impairment, presumably as a result of lowered glucagon levels and other non-insulin related effects [29]. By preventing the degradation of GLP-1 (and GIP), alogliptin restores these endogenous hormones to normal physiological levels and extends their half-lives; thereby assisting in the maintenance of normal plasma glucose levels. The effects of alogliptin are observed predominantly after meals [30, 31].

Alogliptin is rapidly absorbed after oral administration and reaches maximum plasma concentration in approximately 1-2 h. It has a mean half-life of 12-24 h [1, 31]. Alogliptin is only minimally metabolized and is excreted primarily unchanged in the urine (75\%), necessitating dosage adjustments in renal but not hepatic impairment [31]. As alogliptin is neither an inhibitor nor an inducer of cytochrome P450 isoforms, there is a low likelihood of drugdrug interactions [32]. In multiple dose studies (14 days) of alogliptin, plasma DPP-4 activity was inhibited by $>80 \%$ after $24 \mathrm{~h}$, supporting once-daily dosing [31].

\section{Alogliptin-Pioglitazone Combination}

Pharmacokinetic studies in healthy volunteers indicated no clinically significant changes in the area under the curve of the drug concentrationtime profile $\left(\mathrm{AUC}_{0-24 \mathrm{~h}}\right)$, maximum concentration $\left(C_{\max }\right)$, or time to $C_{\max }\left(T_{\max }\right)$ for alogliptin or pioglitazone, or in urine pharmacokinetic parameters for alogliptin, for the combination of alogliptin and pioglitazone compared with each agent administered alone [33].

Animal models of T2DM have demonstrated beneficial and additive effects with the alogliptinpioglitazone combination on several measures of glycemic control compared with either agent alone; thus, providing a strong argument for clinical use of this specific combination. In $o b / o b$ mice and $d b / d b$ mice, combined administration of alogliptin and pioglitazone for approximately 4 weeks significantly improved glycemic control 
and lipid profiles compared with alogliptin or pioglitazone monotherapy [34, 35]. The alogliptin-pioglitazone combination also increased pancreatic insulin content [34-36], maintained islet structure [34], and preserved islet mass [36] to a greater extent than either agent alone.

\section{CLINICAL EVIDENCE FOR ALOGLIPTIN-PIOGLITAZONE COMBINATION}

Several well-controlled studies have investigated alogliptin in combination with pioglitazone in the treatment of T2DM patients with different clinical presentations: alogliptinpioglitazone combination in drug-naïve patients [37]; alogliptin as a second or third "add-on" oral antidiabetic agent in patients being treated with pioglitazone $( \pm$ metformin or sulfonylurea) [38-40]; alogliptin-pioglitazone combination in patients being treated with metformin [41]. All studies were randomized and controlled, with double-blind phases ranging from 12 to 52 weeks in duration. Treatment (either single component or combination) was administered once daily. Patients had baseline $\mathrm{HbA}_{1 \mathrm{c}}$ levels of between $6.9 \%$ and $11 \%$ (mean approximately $8 \%$ in most studies; $8.8 \%$ in drug-naïve patients). The primary efficacy endpoint in all studies was the change in $\mathrm{HbA}_{1 \mathrm{c}}$ from baseline to study end. Secondary efficacy endpoints included changes from baseline in fasting plasma glucose (FPG); proportions of patients achieving specific $\mathrm{HbA}_{1 \mathrm{c}}$ target levels; incidence of hyperglycemic rescue; changes from baseline in markers of beta-cell function and insulin resistance, serum lipids, and body weight. Safety was assessed according to adverse events (AEs), clinical laboratory tests, electrocardiograms, physical examinations, vital signs, and the incidence of hypoglycemia.
In drug-naïve patients with T2DM inadequately controlled by diet and exercise $(n=655)$, alogliptin plus pioglitazone at two different dosage levels (alogliptin $12.5 \mathrm{mg}+$ pioglitazone $30 \mathrm{mg}$ [A12.5 + P30]; alogliptin $25 \mathrm{mg}+$ pioglitazone $30 \mathrm{mg}$ [A25 + P30]) were compared with each individual component as monotherapy (alogliptin $25 \mathrm{mg}$ [A25]; pioglitazone $30 \mathrm{mg}$ [P30]) [37]. For almost all efficacy parameters, A25 + P30 was slightly but consistently superior to A12.5 + P30, and was significantly superior to A25 or P30 alone. The A25 + P30 combination produced a significantly larger reduction from baseline in $\mathrm{HbA}_{1 \mathrm{c}}$ (Table 1) [37-41]. Compared with other treatment regimens, the A25 + P30 combination was also associated with a significantly larger reduction from baseline in FPG $(-2.8 \mathrm{mmol} / \mathrm{L})$, a greater proportion of patients achieving the target $\mathrm{HbA}_{1 \mathrm{c}}$ of $\leq 7.0 \%$ (63\%), and fewer episodes of marked hyperglycemia (25\%). Fewer patients treated with the alogliptinpioglitazone combination at either dose required hyperglycemic rescue during the course of the 26-week trial compared with those receiving alogliptin or pioglitazone monotherapy. Combination therapy was consistently more effective than component monotherapy irrespective of age, gender, race, ethnicity, or body mass index (BMI). Although the incidence of study drug-related adverse effects was highest with the A25 + P30 combination (21.3\%), there was no obvious pattern among treatment arms with respect to the number of serious AEs or treatment discontinuations due to AEs. Mean body weight was increased to a similar extent in groups treated with pioglitazone either as monotherapy or in combination with alogliptin (2.2, 2.5, and $3.1 \mathrm{~kg}$ for P30 alone, A12.5 + P30, and A25 + P30, respectively), whereas it remained virtually unchanged $(-0.3 \mathrm{~kg})$ in the group treated with A25 monotherapy. 
Table 1 Clinical studies of alogliptin-pioglitazone combination treatment in patients with type 2 diabetes mellitus: mean change in $\mathrm{HbA}_{1 \mathrm{c}}$ level from baseline to study end (primary efficacy variable)

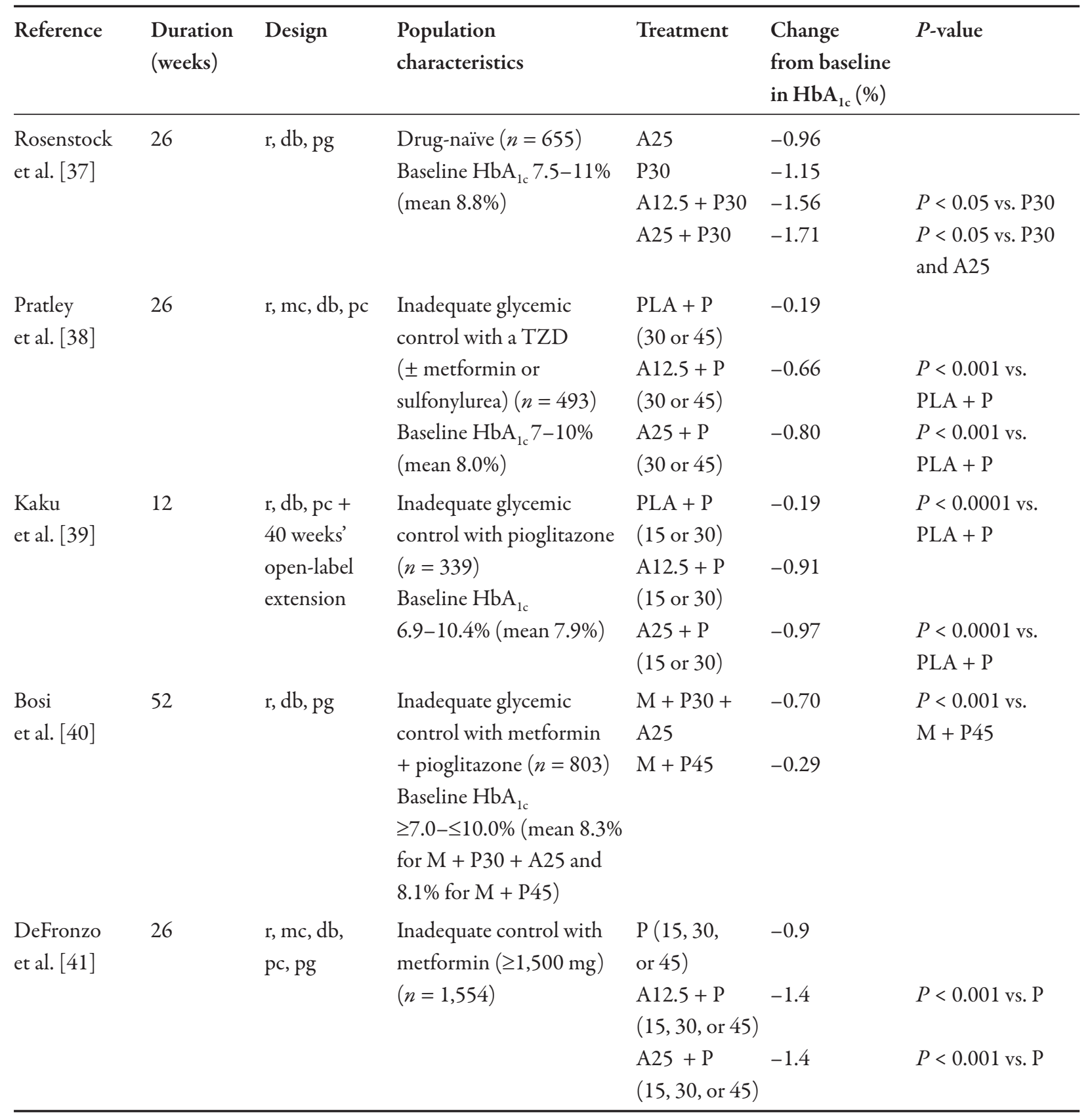

$A$ alogliptin (mg), $d b$ double-blind, $H b A_{l c}$ glycosylated hemoglobin (NGSP value), $M$ metformin, $m c$ multicentre, $P$ pioglitazone (mg), $p c$ placebo-controlled, $p g$ parallel group, $P L A$ placebo, $r$ randomized, $T Z D$ thiazolidinedione

In a large international study encompassing 125 sites in four world regions, the efficacy and safety of alogliptin as add-on therapy was investigated in patients with T2DM $(n=493)$ who had failed to achieve adequate glycemic control with a TZD (pioglitazone or rosiglitazone) with or without concomitant metformin or sulfonylurea [38]. Prior to randomization, patients previously treated with rosiglitazone were switched to an equivalent 
dosage of pioglitazone (P30 or $45 \mathrm{mg}$ ) to which placebo or A12.5 or $25 \mathrm{mg}$ (A25) was added. Patients also taking metformin or sulfonylurea were allowed to continue these medications at the same dosage throughout the study. After 26 weeks' treatment, the mean change from baseline in $\mathrm{HbA}_{1 \mathrm{c}}$ was significantly greater with add-on A12.5 or A25 than with placebo (Table 1); changes during the course of the study were independent of age, BMI, or ethnicity. Significantly more patients treated with either dose of add-on alogliptin achieved an $\mathrm{HbA}_{1 \mathrm{c}}$ of $\leq 7.0 \%(P=0.016)$ and had a reduction in $\mathrm{HbA}_{1 \mathrm{c}}$ of $\geq 1.0 \%(P<0.001)$ compared with their placebo-treated counterparts, whereas significantly fewer alogliptin- than placebotreated patients had marked hyperglycemia $(P<0.001)$. Improvements were statistically significant at the first assessment and at each subsequent measurement throughout the 26-week treatment period. Likewise, FPG levels decreased with add-on A12.5 or A25 within a week of treatment, and remained significantly lower than those produced by placebo throughout the study. At week 26, there were no significant differences between groups in terms of changes from baseline in body weight (increase of approximately $1 \mathrm{~kg}$ in each group) or plasma lipid concentrations. With regard to safety, the incidence of the most common AEs (i.e., those reported by $>5 \%$ of patients) was broadly similar across all three treatment groups. More cardiac disorders occurred in the A25 group than in the A12.5 and placebo groups, but only 2 of 13 reported events were considered possibly drug related and both were of moderate intensity. Overall, a similar proportion of patients (approximately 18\%) in each group experienced at least one treatmentrelated $\mathrm{AE}$ and approximately 3\% in each group discontinued treatment because of an $\mathrm{AE}$ (Fig. 2) [35].

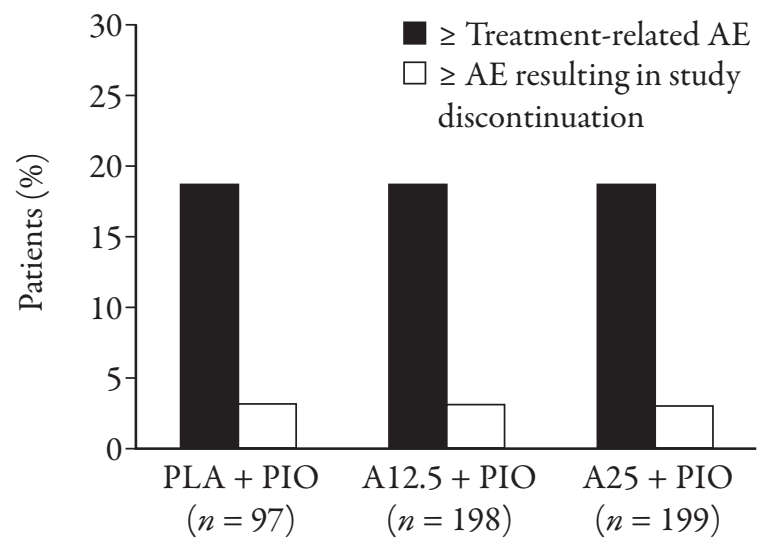

Fig. 2 Incidence of treatment-related AEs and study discontinuations due to AEs in patients with T2DM treated with add-on PLA, A12.5, or A25 to PIO \pm metformin or sulfonylurea [35]. A alogliptin (mg), AE adverse event, PIO pioglitazone, T2DM type 2 diabetes mellitus

The efficacy and safety of add-on alogliptin were also evaluated in a study conducted in Japanese patients with inadequate glycemic control $\left(\mathrm{HbA}_{1 \mathrm{c}}\right.$ 6.9-10.4\%) whilst being treated with pioglitazone and diet/exercise [39]. Patients were initially randomized to receive placebo or A12.5 or A25 in addition to their existing stable dose of pioglitazone $15 \mathrm{mg}$ or P30. After the 12-week double-blind phase, patients could enter a 40-week open-label extension phase intended to assess the combination of alogliptin plus pioglitazone over the longer term. Of 460 enrolled patients, 339 were randomized in the double-blind phase. Similar to the study of Pratley et al. described above [38], both doses of alogliptin produced significant reductions in $\mathrm{HbA}_{1 \mathrm{c}}$ over placebo when added to pioglitazone (Table 1), and these were independent of age, gender, or duration of diabetes. A significantly greater proportion of patients treated with alogliptin than placebo achieved $\mathrm{HbA}_{1 \mathrm{c}}$ levels of $<6.9 \%$ (approximately $50 \%$ with either dose vs. $20 \%$ with placebo). Mean changes from baseline in a range of other glycemic/ metabolic parameters further demonstrated the superiority of add-on alogliptin over placebo (Table 2) [39]. Notably, compared with placebo, 
Table 2 Changes from baseline in several glycemic/metabolic parameters in Japanese patients treated for 12 weeks with addon PLA or alogliptin (A12.5 or A25) to pioglitazone (P15 or P30) [39]

\begin{tabular}{|c|c|c|c|}
\hline & $\begin{array}{l}\mathrm{PLA}+\mathrm{P} \\
(n=115)\end{array}$ & $\begin{array}{c}\mathrm{A} 12.5+\mathrm{P} \\
(n=111)\end{array}$ & $\begin{array}{l}\mathrm{A} 25+\mathrm{P} \\
(n=113)\end{array}$ \\
\hline Fasting plasma glucose (mg/dL) & -2.4 & $-14.9^{*}$ & $-18.9^{*}$ \\
\hline Glycoalbumin (\%) & -0.11 & $-2.73^{*}$ & $-2.79^{*}$ \\
\hline DPP-4 activity $(\mathrm{nmol} / \mathrm{min} / \mathrm{mL})$ & 0.24 & $-9.63^{*}$ & $-10.01^{*}$ \\
\hline HOMA-R & -0.11 & -0.14 & -0.20 \\
\hline HOMA-beta (\%) & 0.06 & $5.48^{*}$ & $6.99 *$ \\
\hline Body weight (kg) & -0.03 & $0.48^{*}$ & $0.46^{*}$ \\
\hline Blood glucose 2-h $(\mathrm{mg} / \mathrm{dL})$ during meal tolerance test & -4.5 & $-31.8^{*}$ & $-41.5^{*}$ \\
\hline Active GLP-1 AUC ${ }_{0-2 \mathrm{~h}}(\mathrm{pmol} \cdot \mathrm{h} / \mathrm{L})$ during meal tolerance test & 0.9 & $10.3^{*}$ & $11.4^{*}$ \\
\hline Total cholesterol (mg/dL) & 0.3 & -4.5 & $-5.7^{*}$ \\
\hline
\end{tabular}

${ }^{*} P<0.05$ versus $P L A+P$

$A$ alogliptin (mg), $A U C_{0-2 h}$ area under the blood glucose concentration time curve from $0 \mathrm{~h}$ to $2 \mathrm{~h}, D P P-4$ dipeptidyl peptidase 4, GLP-1 glucagon-like peptide-1, HOMA-R homeostasis model assessment of insulin resistance, HOMA-beta homeostasis model assessment of beta-cell function, $P$ pioglitazone (mg), $P L A$ placebo

A12.5 or A25 were associated with significantly greater mean changes from baseline in FPG levels, glycoalbumin, proinsulin:insulin ratio, homeostasis model assessment of beta-cell function (HOMA-beta), and serum lipids. Both doses of add-on alogliptin also produced significant decreases in postprandial blood glucose levels and concentrations of active GLP1 , but did not increase the risk of hypoglycemia compared with placebo. The beneficial effects of alogliptin add-on therapy on measures of glycemic control were maintained throughout the 52-week treatment period. Specifically, significant improvements from baseline in HOMA-beta and the proinsulin:insulin ratio, both markers of beta-cell function, were observed at all assessment points in the add-on alogliptin groups. Consistent with its lack of effect on insulin resistance, alogliptin had no significant effect on homeostasis model assessment of insulin resistance (HOMA-R). Add-on alogliptin was generally well tolerated with no dose-related differences in the incidence of adverse effects. During the extension phase, the only drug-related $\mathrm{AE}$ with an incidence of $\geq 3 \%$ was edema (3\% in the A25 group vs. $1.2 \%$ in the A12.5 group), but this was generally mild and is likely to have been related to pioglitazone.

Alogliptin has also been investigated as a third oral antidiabetic drug in patients failing to achieve adequate glycemic control whilst receiving combination treatment with metformin $(\geq 1,500 \mathrm{mg}$ or maximum tolerated dose) and P30 [40]. This study compared the efficacy and safety of adding A25 to a submaximal dose of pioglitazone versus up-titrating the pioglitazone dose to $45 \mathrm{mg}$. As with all other studies described above, greater improvement in measures of glycemic control was noted with the addition of alogliptin. After 52 weeks of treatment, the mean decrease in $\mathrm{HbA}_{1 \mathrm{c}}$ levels was $-0.70 \%$ with metformin $+\mathrm{P} 30+\mathrm{A} 25$ as compared with $-0.29 \%$ in the group treated with metformin and pioglitazone $45 \mathrm{mg}$ (Table 1), and efficacy was independent of gender, age, race, ethnicity, and baseline BMI. Alogliptin add-on therapy produced significantly $(P<0.01)$ greater decreases in FPG at all time points and significantly more patients receiving add-on alogliptin achieved an $\mathrm{HbA}_{1 \mathrm{c}}$ of $\leq 7.0 \%$ compared 
with the pioglitazone up-titration group (33.2\% vs. $21.3 \% ; P<0.001)$. In parallel with the findings of Kaku et al. in Japanese patients [39], significant improvements $(P<0.001)$ in the proinsulin:insulin ratio and HOMA-beta were noted with metformin + P30 + A25 compared with metformin and pioglitazone $45 \mathrm{mg}$ at week 52, whereas no statistically significant differences from baseline were seen between treatment groups for C-peptide concentrations, HOMA-R, and serum lipids. Approximately twice as many patients treated with metformin and pioglitazone $45 \mathrm{mg}$ than with metformin + P30 + A25 (87 vs. 44; $P<0.001$ ) discontinued treatment because of the need for hyperglycemic rescue. The most common drug-related $\mathrm{AE}$ was peripheral edema, which was experienced by eight patients (2.0\%) in the metformin + P30 + A25 group and by 12 patients (3.0\%) in the metformin and pioglitazone $45 \mathrm{mg}$ group.

Lastly, the efficacy and tolerability of alogliptin in combination with pioglitazone were evaluated in patients with T2DM inadequately controlled by maximum doses $(\geq 1,500 \mathrm{mg})$ of metformin monotherapy [41]. The study contained 12 treatment groups: placebo; A12.5 or A25; pioglitazone at doses of 15,30 , or $45 \mathrm{mg}$; and A12.5 and A25 in combination with one of the three doses of pioglitazone. The primary analyses compared pioglitazone monotherapy (all doses pooled, $n=387$ ) with A12.5 plus any dose of pioglitazone (A12.5 + pioglitazone; $n=390$ ) or A25 plus any dose of pioglitazone (A25 + pioglitazone; $n=390)$. Combined with any dose of pioglitazone, both doses of alogliptin produced durable reductions in $\mathrm{HbA}_{1 \mathrm{c}}$ that were greater than those produced by any dose of pioglitazone alone. At week 26, the change from baseline in $\mathrm{HbA}_{1 \mathrm{c}}$ was $-1.4 \%$ for both A12.5 + pioglitazone and A25 + pioglitazone, compared with $-0.9 \%$ for pioglitazone alone (both comparisons $P<0.001$ ) (Table 1).
Again, no differences in efficacy were observed for any predefined patient subgroup based on gender, age, race, ethnicity, or baseline BMI. Approximately $55 \%$ of patients treated with the alogliptin and pioglitazone in combination achieved an $\mathrm{HbA}_{1 \mathrm{c}}$ of $\leq 7.0 \%$. The alogliptinpioglitazone combination also produced greater reductions in FPG $(-2.5 \mathrm{mmol} / \mathrm{L}$ for both alogliptin doses) than pioglitazone alone $(-1.6 \mathrm{mmol} / \mathrm{L} ; P<0.001)$, and fewer patients receiving the combination therapy required hyperglycemic rescue $(1.5-4.6 \%)$ than those treated with either component alone (8.5-14.7\%) or placebo (31.8\%). Both A12.5 + pioglitazone and for A25 + pioglitazone produced significantly greater improvement in measures of beta-cell function (proinsulin:insulin ratio and HOMAbeta) than pioglitazone alone, but had no obvious additive effect on HOMA-R. There was a weight gain of between $1.5 \mathrm{~kg}$ and $1.9 \mathrm{~kg}$ in groups treated with pioglitazone either as monotherapy or in combination, and alogliptin neither prevented nor exacerbated this weight gain. All regimens were well tolerated; hypoglycemia was uncommon and generally mild-to-moderate in severity, although two severe cases were reported when alogliptin was added to pioglitazone and metformin [40]. Relative to pioglitazone monotherapy, alogliptin-pioglitazone combination therapy was not associated with any meaningful differences in weight gain, incidence of hypoglycemia, AEs, serious AEs, or treatment discontinuations due to an $\mathrm{AE}$.

\section{DISCUSSION}

The treatment of T2DM has long encompassed a stepwise approach beginning with diet and exercise and an oral antihyperglycemic agent, followed by the addition of one or more agents as required in the event that glycemic control deteriorates. This approach is reactive 
rather than proactive as it is based around compensating for the progressive decline of betacell function that underlies the deterioration in glycemic control. It is now known that beta-cell failure occurs much earlier and is more severe than previously thought ( $>80 \%$ failure at the time of diagnosis) [22], suggesting that a newer approach to T2DM treatment is warranted. In a Banting Lecture [23], DeFronzo called for a paradigm shift in the management of T2DM based on the following principles.

1. Effective treatment of $\mathrm{T} 2 \mathrm{DM}$ requires multiple drugs used in combination to correct multiple pathophysiological defects.

2. Treatment should be based on known pathogenic abnormalities and not simply on reduction of $\mathrm{HbA}_{1 \mathrm{c}}$.

3. Therapy must be started early in the natural history of T2DM to prevent progressive beta-cell failure.

The combination of alogliptin and pioglitazone appears to address these more modern requirements for treatment of T2DM as these agents work by complementary mechanisms; alogliptin increases insulin secretion and decreases glucagon secretion after meals $[11,12]$, whereas pioglitazone increases both peripheral and hepatic insulin sensitivity [13]. In an animal model of T2DM, the combination of alogliptin and pioglitazone administered at an early stage of diabetes was shown to improve beta-cell function and maintain islet structure to a greater extent than either component as monotherapy [34].

Randomized controlled trials in which combination therapy with alogliptin and pioglitazone was investigated clinically in patients with T2DM provide strong and consistent clinical evidence for the efficacy of this particular combination. In these trials, alogliptin combined with pioglitazone was evaluated within the framework of several typical T2DM treatment backgrounds: alogliptin-pioglitazone as initial therapy in drug-naïve patients [37]; alogliptin as add-on treatment to pioglitazone $( \pm$ metformin or sulfonylurea) [38, 39]; alogliptin as add-on treatment to pioglitazone + metformin [40]; and alogliptin-pioglitazone as add-on treatment to metformin [41]. The double-blind phase of these studies ranged from 12 to 52 weeks in duration, providing a reasonable estimate of the safety and tolerability of the combination over a longer term.

Irrespective of patients' previous and current exposure to antidiabetic drugs, alogliptin and pioglitazone given in combination was superior to any other study regimen as regards the reduction in $\mathrm{HbA}_{1 \mathrm{c}}$ levels (primary efficacy measure) and secondary measures of glycemic control. From a typical mean baseline $\mathrm{HbA}_{1 \mathrm{c}}$ of approximately $8.0 \%$, the alogliptin and pioglitazone in combination produced statistically significant reductions of between $0.6 \%$ and $1.0 \%$ over and above those produced by either agent alone ( \pm metformin or sulfonylurea). In parallel, significantly greater proportions of patients treated with combination therapy achieved target $\mathrm{HbA}_{1 \mathrm{c}}$ levels as defined per study (generally $\leq 7.0 \%$ ). In all studies, responder analyses according to prespecified subgroups showed no differences in efficacy based on age, gender, baseline BMI, duration of diabetes, or ethnicity, indicating that the combination can be used across the T2DM patient spectrum. All studies reported significantly greater reductions in FPG with alogliptin-pioglitazone combination therapy. Importantly, the combination of alogliptin and pioglitazone $( \pm$ another antidiabetic agent) consistently produced greater improvements in measures of beta-cell function (i.e., HOMA-beta function and proinsulin:insulin ratio) than study regimens in which these two agents were not combined [38-40], suggestive of an additive effect. 
Across all studies, the alogliptin and pioglitazone combination therapy was well tolerated. No new safety issues emerged with the combination outside of the known or potential adverse effects of each individual component. Hypoglycemia was uncommon and generally mild-to-moderate in severity, although two severe cases were reported when alogliptin was added to pioglitazone and metformin [40]. Consistent with the known adverse effects of TZD, pioglitazone was associated with an increase in body weight $[37,38,40]$ but alogliptin appeared to be weight-neutral as it neither prevented nor exacerbated weight gain.

It can be concluded from these results that the alogliptin-pioglitazone combination successfully meets the more modern requirements for treatment of T2DM as outlined above [23] and represents a rational approach to the treatment of T2DM. The complementary mechanisms of action of alogliptin and pioglitazone address several of the underlying metabolic abnormalities in patients with T2DM. The efficacy of this specific combination extends beyond that of reducing $\mathrm{HbA}_{1 \mathrm{c}}$ levels to include improvements in several other measures of glycemic control. Importantly, the ability of the alogliptin-pioglitazone combination to preserve beta-cell function offers a degree of promise in terms of slowing or halting the progression of disease. Should this be confirmed with longer-term use, it is anticipated that alogliptin-pioglitazone combination treatment may offer value when given early in the course of T2DM. The clinical benefits of combining a DPP-4 inhibitor with pioglitazone for improving glycemic control in patients with T2DM has also been documented for other agents in this class, such as vildagliptin [42], sitagliptin [43-45], saxagliptin [46], and linagliptin [47].

The introduction of the fixed-dose combination alogliptin and pioglitazone in Japan is relatively recent and future investigations should investigate its clinical efficacy and safety relative to other combinations of antidiabetic drugs in patients with T2DM. Given the high rate of co-existing medical conditions in patients with T2DM it is also important to assess the impact of the combination on clinical outcomes, including cardiovascular outcomes in patients at risk. In this regard, the Examination of cArdiovascular outcoMes with alogliptin versus standard carE in patients with type 2 diabetes mellitus and acute coronary syndrome (EXAMINE) study is planned to include $>5,000$ men and women with T2DM and acute coronary syndrome, and who will receive alogliptin or placebo for up to 4.5 years in a well-controlled randomized trial [48].

Simplification of drug administration via the convenience of an alogliptin-pioglitazone fixed-dose combination tablet provides the opportunity to improve compliance over that which can be achieved by concomitant treatment with the individual constituents and while maximally exerting the intended antihyperglycemic effect; thereby potentially enhancing treatment efficacy and clinical outcomes.

\section{ACKNOWLEDGMENTS}

The authors thank the Takeda Pharmaceutical Company, Ltd for assistance with literature retrieval, and for funding the editorial assistance provided by Kerry Dechant, Content Ed Net.

Dr. Kaku is the guarantor for this article, and takes responsibility for the integrity of the work as a whole.

Conflicts of Interest. Dr. Kaku was an independent medical expert for clinical studies regarding alogliptin-pioglitazone combination therapy and the alogliptin-pioglitazone fixeddosed combination. Dr. Kaku also interpreted 
the clinical findings and contributed to the discussion and writing of the manuscript. Dr. Hirayama and Dr. Komura were responsible for organizational issues and data analyses of these clinical studies in Japan. All authors contributed to the preparation and/or review of the manuscript with full access to all the clinical data.

Open Access. This article is distributed under the terms of the Creative Commons Attribution Noncommercial License which permits any noncommercial use, distribution, and reproduction in any medium, provided the original author(s) and source are credited.

\section{REFERENCES}

1. Andukuri R, Drincic A, Rendell M. Alogliptin: a new addition to the class of DPP-4 inhibitors. Diabetes Metab Syndr Obes. 2009;2:117-26.

2. Ford ES, Li C, Little RR, Mokdada AH. Trends in A1C concentrations among US adults with diagnosed diabetes from 1999 to 2004. Diabetes Care. 2008;31:102-4.

3. Kobayashi M, Yamazaki K, Hirao K, et al. The status of diabetes control and antidiabetic drug therapy in Japan--a cross-sectional survey of 17,000 patients with diabetes mellitus (JDDM 1). Diabetes Res Clin Pract. 2006;73:198-204.

4. Bron $\mathrm{M}$, Marynchenko $\mathrm{M}$, Yang $\mathrm{H}, \mathrm{Yu} \mathrm{AP}, \mathrm{Wu}$ EQ. Hypoglycemia, treatment discontinuation, and costs in patients with type 2 diabetes mellitus on oral antidiabetic drugs. Postgrad Med. 2012;124:124-32.

5. Japan Diabetes Society. Treatment Guide for Diabetes. Tokyo: Japan Diabetes Society; 2012-2013.

6. Cramer JA. A systematic review of adherence with medications for diabetes. Diabetes Care. 2004;27:1218-24.

7. Pladevall M, Williams LK, Potts LA, Divine G, Xi $\mathrm{H}$, Lafata JE. Clinical outcomes and adherence to medications measured by claims data in patients with diabetes. Diabetes Care. 2004;27:2800-5.

8. Schectman JM, Nadkarni MM, Voss JD. The association between diabetes metabolic control and drug adherence in an indigent population. Diabetes Care. 2002;25:1015-21.
9. Dailey G, Kim MS, Lian JF. Patient compliance and persistence with antihyperglycemic drug regimens: evaluation of a Medicaid patient population with type 2 diabetes mellitus. Clin Ther. 2001;23:131120.

10. Melikian C, White TJ, Vanderplas A, Dezii CM, Chang E. Adherence to oral antidiabetic therapy in a managed care organization: a comparison of monotherapy, combination therapy, and fixed-dose combination therapy. Clin Ther. 2002;24:460-7.

11. Christopher R, Karim A. Clinical pharmacology of alogliptin, a dipeptidyl peptidase- 4 inhibitor, for the treatment of Type 2 diabetes. Expert Rev Clin Pharmacol. 2009;2:589-600.

12. Derosa G, Maffioli P. Dipeptidyl peptidase-4 inhibitors: 3 years of experience. Diabetes Technol Ther. 2012;14:350-64.

13. Yki-Järvinen H. Thiazolidinediones. N Engl J Med. 2004;351:1106-18.

14. Derosa G, Maffioli P. Peroxisome proliferatoractivated receptor- $\gamma$ (PPAR- $\gamma$ ) agonists on glycemic control, lipid profile and cardiovascular risk. Curr Mol Pharmacol. 2012;5:272-81.

15. Triplitt C, Cersosimo E, DeFronzo RA. Pioglitazone and alogliptin combination therapy in type 2 diabetes: a pathophysiologically sound treatment. Vasc Health Risk Manag. 2010;6:671-90.

16. Miyazaki Y, Mahankali A, Wajcberg E, Bajaj M, Mandarino LJ, DeFronzo RA. Effect of pioglitazone on circulating adipocytokine levels and insulin sensitivity in type 2 diabetic patients. J Clin Endocrinol Metab. 2004;89:4312-9.

17. Bodles AM, Banga A, Rasouli N, Ono F, Kern PA, Owens RJ. Pioglitazone increases secretion of highmolecular-weight adiponectin from adipocytes. Am J Physiol Endocrinol Metab. 2006;291:E1100-5.

18. Charbonnel B, Schernthaner G, Brunetti P, et al. Long-term efficacy and tolerability of addon pioglitazone therapy to failing monotherapy compared with addition of gliclazide or metformin in patients with type 2 diabetes. Diabetologia. 2005;48:1093-104.

19. Hanefeld M, Pfützner A, Forst T, Lübben G. Glycemic control and treatment failure with pioglitazone versus glibenclamide in type 2 diabetes mellitus: a 42-month, open-label, observational, primary care study. Curr Med Res Opin. 2006;22:1211-5. 
20. Mazzone T, Meyer PM, Feinstein SB, et al. Effect of pioglitazone compared with glimepiride on carotid intima-media thickness in type 2 diabetes: a randomized trial. JAMA. 2006;296:2572-81.

21. Nissen SE, Nicholls SJ, Wolski K, et al. Comparison of pioglitazone vs glimepiride on progression of coronary atherosclerosis in patients with type 2 diabetes: the PERISCOPE randomized controlled trial. JAMA. 2008;299:1561-73.

22. Tan MH, Baksi A, Krahulec B, et al. Comparison of pioglitazone and gliclazide in sustaining glycemic control over 2 years in patients with type 2 diabetes. Diabetes Care. 2005;28:544-50.

23. DeFronzo RA. Banting Lecture. From the triumvirate to the ominous octet: a new paradigm for the treatment of type 2 diabetes mellitus. Diabetes. 2009;58:773-95.

24. Derosa G, Mereu R, D'Angelo A, et al. Effect of pioglitazone and acarbose on endothelial inflammation biomarkers during oral glucose tolerance test in diabetic patients treated with sulphonylureas and metformin. J Clin Pharm Ther. 2010;35:565-79.

25. Derosa G, Cicero AF, Fogari E, D'Angelo A, Bianchi L, Maffioli P. Pioglitazone compared to glibenclamide on lipid profile and inflammation markers in type 2 diabetic patients during an oral fat load. Horm Metab Res. 2011;43:505-12.

26. Hanefeld M. Pharmacokinetics and clinical efficacy of pioglitazone. Int J Clin Pract Suppl. 2001;121:19-25.

27. Nowak SN, Edwards DJ, Clarke A, Anderson GD, Jaber LA. Pioglitazone: effect on CYP3A4 activity. J Clin Pharmacol. 2002;42:1299-302.

28. Scheen AJ. Pharmacokinetic interactions with thiazolidinediones. Clin Pharmacokinet. 2007;46:1-12.

29. Dupre J. Glycaemic effects of incretins in type 1 diabetes mellitus: a concise review, with emphasis on studies in humans. Regul Pept. 2005;128:149-57.

30. Mikhail N. Combination therapy with DPP-4 inhibitors and pioglitazone in type 2 diabetes: theoretical consideration and therapeutic potential. Vasc Health Risk Manag. 2008;4:1221-7.

31. Pratley RE. Alogliptin: a new, highly selective dipeptidyl peptidase- 4 inhibitor for the treatment of type 2 diabetes. Expert Opin Pharmacother. 2009;10:503-12.
32. Scheen AJ. Dipeptidylpeptidase-4 inhibitors (gliptins): focus on drug-drug interactions. Clin Pharmacokinet. 2010;49:573-88.

33. Karim A, Laurent A, Munsaka M, Wann E, Fleck $\mathrm{P}$, Mekki Q. Coadministration of pioglitazone or glyburide and alogliptin: pharmacokinetic drug interaction assessment in healthy participants. J Clin Pharmacol. 2009;49:1210-9.

34. Moritoh Y, Takeuchi K, Asakawa T, Kataoka O, Odaka H. Combining a dipeptidyl peptidase-4 inhibitor, alogliptin, with pioglitazone improves glycaemic control, lipid profiles and betacell function in $\mathrm{db} / \mathrm{db}$ mice. $\mathrm{Br} \mathrm{J}$ Pharmacol. 2009;157:415-26.

35. Moritoh Y, Takeuchi K, Asakawa T, Kataoka O, Odaka $H$. The dipeptidyl peptidase-4 inhibitor alogliptin in combination with pioglitazone improves glycemic control, lipid profiles, and increases pancreatic insulin content in ob/ob mice. Eur J Pharmacol. 2009;602:448-54.

36. Kawashima S, Matsuoka TA, Kaneto $\mathrm{H}$, et al. Effect of alogliptin, pioglitazone and glargine on pancreatic $\beta$-cells in diabetic $\mathrm{db} / \mathrm{db}$ mice. Biochem Biophys Res Commun. 2011;404:534-40.

37. Rosenstock J, Inzucchi SE, Seufert J, Fleck PR, Wilson CA, Mekki Q. Initial combination therapy with alogliptin and pioglitazone in drug-naïve patients with type 2 diabetes. Diabetes Care. 2010;33:2406-8.

38. Pratley RE, Reusch JE, Fleck PR, Wilson CA, Mekki Q; Alogliptin Study 009 Group. Efficacy and safety of the dipeptidyl peptidase- 4 inhibitor alogliptin added to pioglitazone in patients with type 2 diabetes: a randomized, double-blind, placebo-controlled study. Curr Med Res Opin. 2009;25:2361-71.

39. Kaku K, Itayasu T, Hiroi S, Hirayama M, Seino Y. Efficacy and safety of alogliptin added to pioglitazone in Japanese patients with type 2 diabetes: a randomized, double-blind, placebocontrolled trial with an open-label longterm extension study. Diabetes Obes Metab. 2011;13:1028-35.

40. Bosi E, Ellis GC, Wilson CA, Fleck PR. Alogliptin as a third oral antidiabetic drug in patients with type 2 diabetes and inadequate glycaemic control on metformin and pioglitazone: a 52-week, randomized, double-blind, active-controlled, parallel-group study. Diabetes Obes Metab. 2011;13:1088-96. 
41. DeFronzo RA, Burant CF, Fleck P, Wilson C, Mekki Q, Pratley RE. Efficacy and tolerability of the DPP4 inhibitor alogliptin combined with pioglitazone, in metformin-treated patients with type 2 diabetes. J Clin Endocrinol Metab. 2012;97:1615-22.

42. Derosa G, Maffioli P, Ferrari I, et al. Effects of one year treatment of vildagliptin added to pioglitazone or glimepiride in poorly controlled type 2 diabetic patients. Horm Metab Res. 2010;42:663-9.

43. Derosa G, Maffioli P, Salvadeo SA, et al. Effects of sitagliptin or metformin added to pioglitazone monotherapy in poorly controlled type 2 diabetes mellitus patients. Metabolism. 2010;59:887-95.

44. Rosenstock J, Brazg R, Andryuk PJ; the Sitagliptin Study 019 Group. Efficacy and safety of the dipeptidyl peptidase-4 inhibitor sitagliptin added to ongoing pioglitazone therapy in patients with type 2 diabetes: a 24-week, multicentre, randomized, double-blind, placebo-controlled, parallel-group study. Clin Ther. 2006;28:1556-68.

45. Yoon KH, Steinberg H, Teng R, et al. Efficacy and safety of initial combination therapy with sitagliptin and pioglitazone in patients with type 2 diabetes: a 54-week study. Diabetes Obes Metab. 2012;14:745-52.

46. Hollander PL, Li J, Frederich R; the CV181013 Investigators. Safety and efficacy of saxagliptin added to thiazolidinedione over 76 weeks in patients with type 2 diabetes mellitus. Diabetes Vasc Dis Res. 2011;8:125-35.

47. Gomis R, Espadero R-M, Jones R, Dugi KA. Efficacy and safety of initial combination therapy with linagliptin and pioglitazone in patients with inadequately controlled type 2 diabetes: a randomized, double-blind, placebo-controlled study. Diabetes Obes Metab. 2011;13:653-61

48. White WB, Bakris GL, Bergenstal RM, et al. Examination of cArdiovascular outcoMes with alogliptin versus standard carE in patients with type 2 diabetes mellitus and acute coronary syndrome (EXAMINE): a cardiovascular safety study of the dipeptidyl peptidase 4 inhibitor alogliptin in patients with type 2 diabetes with acute coronary syndrome. Am Heart J. 2011;162:620-6. 